PROCEEDINGS OF THE

AMERICAN MATHEMATICAL SOCIETY

Volume 128, Number 4, Pages 1157-1166

S 0002-9939(99)05198-9

Article electronically published on August 17, 1999

\title{
COMPLETENESS IN THE SET OF WAVELETS
}

\author{
GUSTAVO GARRIGÓS AND DARRIN SPEEGLE \\ (Communicated by David R. Larson)
}

\begin{abstract}
We study the completeness properties of the set of wavelets in $L^{2}(\mathbb{R})$. It is well-known that this set is not closed in the unit ball of $L^{2}(\mathbb{R})$. However, if one considers the metric inherited as a subspace (in the Fourier transform side) of $L^{2}(\mathbb{R}, d \xi) \cap L^{2}\left(\mathbb{R}_{*}, \frac{d \xi}{|\xi|}\right)$, we do obtain a complete metric space.
\end{abstract}

\section{IntRoduction}

An orthonormal wavelet is a function $\psi \in L^{2}(\mathbb{R})$ such that the system

$$
\psi_{j, k}(x)=2^{j / 2} \psi\left(2^{j} x-k\right), \quad j, k \in \mathbb{Z},
$$

forms an orthonormal basis for $L^{2}(\mathbb{R})$. We shall denote the set of all wavelets by $\mathcal{W}$. Note that, by definition, $\mathcal{W}$ is a subset of the unit sphere of $L^{2}(\mathbb{R})$.

Many important properties of wavelets can be seen by looking at their Fourier transforms. In this paper, we shall denote the Fourier transform of a function $f \in L^{1}(\mathbb{R})$ by

$$
\hat{f}(\xi)=\int_{\mathbb{R}} f(x) e^{-i x \xi} d x, \quad \xi \in \mathbb{R} .
$$

If $f \in L^{2}(\mathbb{R}), \hat{f}$ will denote the extension of the densely defined operator in (1.2) from $L^{1}(\mathbb{R}) \cap L^{2}(\mathbb{R})$ to $L^{2}(\mathbb{R})$. Note that, in this case, the inverse Fourier transform of a function $f \in L^{1}(\mathbb{R}) \cap L^{2}(\mathbb{R})$ is given by

$$
\check{f}(x)=\frac{1}{2 \pi} \int_{\mathbb{R}} f(\xi) e^{i x \xi} d \xi, \quad x \in \mathbb{R} .
$$

The set of all the Fourier transforms of functions in $\mathcal{W}$ will be denoted by $\widehat{\mathcal{W}}$. That is,

$$
\widehat{\mathcal{W}}=\left\{\hat{\psi} \in L^{2}(\mathbb{R}): \psi \text { is a wavelet }\right\} .
$$

It is well-known that, by means of their Fourier transforms, wavelets can be characterized as the functions $\hat{\psi}$ in the sphere of radius $\sqrt{2 \pi}$ of $L^{2}(\mathbb{R}, d \xi)$ satisfying the equations

$$
\delta_{\hat{\psi}}(\xi) \equiv \sum_{j \in \mathbb{Z}}\left|\hat{\psi}\left(2^{j} \xi\right)\right|^{2}=1, \quad \text { a.e. } \xi \in \mathbb{R}
$$

Received by the editors June 15, 1998.

1991 Mathematics Subject Classification. Primary 42C15.

Key words and phrases. Wavelets, completeness, equivalent metrics.

(C)2000 American Mathematical Society 


$$
t_{q, \hat{\psi}}(\xi) \equiv \sum_{j=0}^{\infty} \hat{\psi}\left(2^{j} \xi\right) \overline{\hat{\psi}\left(2^{j}(\xi+2 q \pi)\right)}=0, \quad \text { a.e. } \xi \in \mathbb{R}, \quad \forall q \in 2 \mathbb{Z}+1 .
$$

(See Theorem 1.1 in Chapter 7 of [HW].)

In addition, every function $\psi$ generating an orthonormal system as in (1.1) must satisfy the equations:

$$
\begin{gathered}
\tau_{\hat{\psi}}(\xi)=\sum_{k \in \mathbb{Z}}|\hat{\psi}(\xi+2 \pi k)|^{2}=1, \quad \text { a.e. } \xi \in \mathbb{R}, \\
\tau_{j, \hat{\psi}}(\xi)=\sum_{k \in \mathbb{Z}} \hat{\psi}\left(2^{j}(\xi+2 \pi k)\right) \overline{\hat{\psi}(\xi+2 k \pi)}=0, \quad \text { a.e. } \xi \in \mathbb{R}, \quad j \geq 1 .
\end{gathered}
$$

(See (1.1) and (1.2) in Chapter 3 of [HW].)

The characterizations above suggest that the set $\widehat{\mathcal{W}}$ might be a closed topological subspace of the $\sqrt{2 \pi}$-sphere of $L^{2}(\mathbb{R}, d \xi)$. However, the situation is a little more subtle.

To describe better some of the problems arising, we introduce a particularly simple class of wavelets: $\mathcal{W}_{s}$. These are the wavelets whose Fourier transform is the characteristic function of a measurable subset of $\mathbb{R}$; that is,

$$
\widehat{\mathcal{W}}_{s}=\left\{\chi_{K}: K \text { is measurable and } \chi_{K} \in \widehat{\mathcal{W}}\right\} .
$$

The terms: "s-elementary" [SPEE], unimodular [HKLS] or MSF ([FW], [HW]) are used in the literature to refer to these wavelets. The characterization theorem for elements in $\mathcal{W}_{s}$ has a simpler form than in the general case: a function $\hat{\psi}=$ $\chi_{K} \in L^{2}(\mathbb{R})$ belongs to $\widehat{\mathcal{W}}_{s}$ if and only if satisfies the equations (1.5) and (1.7); equivalently, if there is a partition $\left\{K_{\ell}\right\}_{\ell \in \mathbb{Z}}$ of $K$ such that $\left\{K_{\ell}+2 \ell \pi\right\},\left\{2^{\ell} K_{\ell}\right\}$, $\ell \in \mathbb{Z}$, are each a partition of $\mathbb{R}$ (see Theorem 2.3 and Corollary 2.4 in Chapter 7 of [HW]). One simple example of a wavelet in $\mathcal{W}_{s}$ is the Shannon wavelet, whose Fourier transform is given by $\hat{\psi}=\chi_{[-2 \pi,-\pi) \cup[\pi, 2 \pi)}$.

Going back to our original question, Example 1 below, taken from [HKLS], shows that $\widehat{\mathcal{W}}_{s}$ is not closed when considered as a subspace of $L^{2}(\mathbb{R}, d \xi)$. Since $\widehat{\mathcal{W}}_{s}$ is closed in $\widehat{\mathcal{W}}$ (with the topology induced by $L^{2}(\mathbb{R})$ ), this implies that $\widehat{\mathcal{W}}$ is not closed in $L^{2}(\mathbb{R}, d \xi)$, either. In particular, $\widehat{\mathcal{W}}$, with the metric inherited from $L^{2}(\mathbb{R}, d \xi)$,

$$
d_{1}(f, g)=\|f-g\|_{L^{2}(\mathbb{R}, d \xi)}=\left(\int_{\mathbb{R}}|f(\xi)-g(\xi)|^{2} d \xi\right)^{1 / 2}, \quad f, g \in L^{2}(\mathbb{R}, d \xi)
$$

is not a complete metric space.

Example 1. A collection of MSF wavelets which converges (in $L^{2}(\mathbb{R})$ ) to a function which is not a wavelet.

Consider the family of functions $\hat{\psi}_{a}=\chi_{[-2 a,-a) \cup[2 \pi-a, 4 \pi-2 a)}$, for $0<a \leq \pi$. It is straightforward to check that $\hat{\psi}_{a}$ satisfies (1.5) and (1.7), and therefore, $\hat{\psi}_{a} \in \widehat{\mathcal{W}}_{s}$. However, if $\left(a_{n}\right)_{n=1}^{\infty}$ is any sequence in $(0, \pi]$ converging to 0 , then by the dominated convergence theorem,

$$
\int_{\mathbb{R}}\left|\hat{\psi}_{a_{n}}(\xi)-\chi_{[2 \pi, 4 \pi)}(\xi)\right|^{2} d \xi \rightarrow 0, \quad \text { as } n \rightarrow \infty .
$$


But, the limit function $\hat{\psi}=\chi_{[2 \pi, 4 \pi)}$ does not satisfy (1.5). Therefore, $\hat{\psi} \notin \widehat{\mathcal{W}}_{s}$, and $\widehat{\mathcal{W}}_{s}$ is not closed in $L^{2}(\mathbb{R}, d \xi)$.

We may consider another "natural" metric on $\widehat{\mathcal{W}}$; the metric $d_{2}$ inherited as a subspace of $L^{2}\left(\mathbb{R}_{*},|d \xi|\right)$, namely,

$$
d_{2}(f, g)=\left(\int_{\mathbb{R}_{*}}|f(\xi)-g(\xi)|^{2} \frac{d \xi}{|\xi|}\right)^{1 / 2}, \quad f, g \in L^{2}\left(\mathbb{R}_{*}, \mid \xi \xi\right) .
$$

Here, we are regarding $\left(\mathbb{R}_{*}, \frac{d \xi}{|\xi|}\right)$ as the measure space $(\mathbb{R} \backslash\{0\}, \mathcal{L}, \mu)$, where $\mathcal{L}$ are the Lebesgue measurable sets in $\mathbb{R}_{*}=\mathbb{R} \backslash\{0\}$ and

$$
\mu(E)=\int_{E} \frac{1}{|x|} d \xi, \quad E \in \mathcal{L}
$$

Now, it is easy to check that if $\hat{\psi} \in \widehat{\mathcal{W}}$, then $\hat{\psi}$ lies in a sphere of radius $\sqrt{\ln (4)}$ in $L^{2}\left(\mathbb{R}_{*}, \mid \begin{array}{l}d \xi \\ \mid \xi\end{array}\right)$. Indeed,

$$
\begin{aligned}
\int_{\mathbb{R}_{*}}|\hat{\psi}(\xi)|^{2} \frac{d \xi}{|\xi|} & =\sum_{j \in \mathbb{Z}} \int_{2^{j} \leq|\xi|<2^{j+1}}|\hat{\psi}(\xi)|^{2} \frac{d \xi}{|\xi|} \\
& =\int_{1 \leq|\xi|<2}\left(\sum_{j \in \mathbb{Z}}\left|\hat{\psi}\left(2^{-j} \xi\right)\right|^{2}\right) \frac{d \xi}{|\xi|} \\
& =2 \int_{1}^{2} \frac{d \xi}{\xi}=\ln (4),
\end{aligned}
$$

where the third equality follows from (1.5). is,

We will denote by $d$ the metric in $L^{2}(\mathbb{R}, d \xi) \cap L^{2}\left(\mathbb{R}_{*},|\xi| \xi \mid\right)$ given by $d_{1}+d_{2}$. That

$$
d(f, g)=d_{1}(f, g)+d_{2}(f, g), \quad f, g \in L^{2}(\mathbb{R}, d \xi) \cap L^{2}\left(\mathbb{R}_{*}, \mid \begin{array}{l}
d \xi \\
\mid \xi
\end{array}\right)
$$

The main result in this paper is the following:

Theorem 1.13. The metrics $d_{1}, d_{2}$ and $d$ are topologically equivalent on the set $\widehat{\mathcal{W}}$. Moreover, $(\widehat{\mathcal{W}}, d)$ is a complete metric space.

We saw in Example 1 above that $\left(\widehat{\mathcal{W}}, d_{1}\right)$ is not complete. However, this statement does not contradict Theorem 1.13 because the sequence $\left\{\hat{\psi}_{a_{n}}\right\}_{n=1}^{\infty}$ is not $d_{2}$-Cauchy. Indeed, if $0<2 b<a \leq \pi$, then

$$
\begin{aligned}
\int_{\mathbb{R}_{*}}\left|\hat{\psi}_{a}(\xi)-\hat{\psi}_{b}(\xi)\right|^{2} \frac{d \xi}{|\xi|} & \geq \int_{-\infty}^{0}\left|\chi_{[-2 a,-a)}(\xi)-\chi_{[-2 b,-b)}(\xi)\right|^{2} \frac{d \xi}{|\xi|} \\
& =\int_{a}^{2 a} \stackrel{d \xi}{\xi}+\int_{b}^{2 b} \stackrel{d \xi}{\xi}=\ln (4) .
\end{aligned}
$$

Before proving Theorem 1.13, we give an example which shows that $\left(\widehat{\mathcal{W}}, d_{2}\right)$ is not a complete metric space either.

Example 2. $\left(\widehat{\mathcal{W}}, d_{2}\right)$ is not complete.

\footnotetext{
${ }^{1}$ Since $\{0\}$ is a null set in $(\mathbb{R}, \mathcal{L}, d \xi)$, we may identify the spaces $L^{2}(\mathbb{R}, d \xi) \equiv L^{2}\left(\mathbb{R}_{*}, d \xi\right)$. Then, the intersection $L^{2}(\mathbb{R}, d \xi) \cap L^{2}\left(\mathbb{R}_{*},|\xi \xi|\right)$ may be regarded as the space $L^{2}\left(\mathbb{R}_{*},\left(1+\frac{1}{|\xi|}\right) d \xi\right)$.
} 


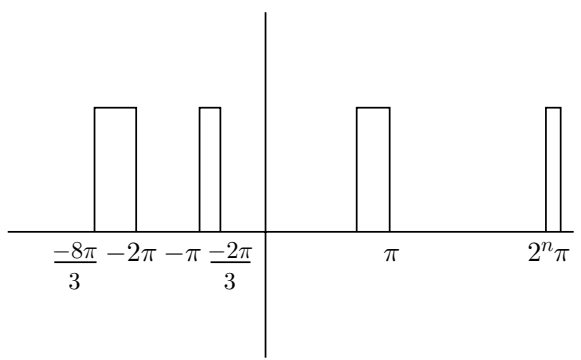

\section{Diagram 1.}

We will exhibit a $d_{2}$-Cauchy sequence in $\widehat{\mathcal{W}}_{s}$ whose $d_{2}$-limit point does not belong to $\widehat{\mathcal{W}}_{s}$. This is a particular example of the wavelets constructed by Fang and Wang (see Example 5 of [FW]). Let, for $n \geq 1$,

$$
K_{n}=\left[-\frac{8 \pi}{3},-2 \pi\right) \cup\left[-\pi,-\frac{2 \pi,}{3}\right) \cup\left[\frac{2^{n} \pi}{2^{n+1}-1}, \pi\right) \cup\left[2^{n} \pi, 2^{n} \pi+\frac{2^{n} \pi}{2^{n+1}-1}\right)
$$

(see Diagram 1). Then, $\hat{\psi}=\chi_{K_{n}} \in \widehat{\mathcal{W}}_{s}$ for every $n \geq 1$. Indeed, this can be easily verified by checking that equations (1.5) and (1.7) hold.

We claim that $\lim _{n \rightarrow \infty} d_{2}\left(\hat{\psi}_{n}, \hat{\psi}\right)=0$, where $\hat{\psi}=\chi_{K}$, and $K=\left[-\frac{8 \pi}{3},-2 \pi\right) \cup$ $\left[-\pi,-\frac{2 \pi}{3}\right) \cup\left(\frac{\pi}{2}, \pi\right)$. Note that $\hat{\psi} \notin \widehat{\mathcal{W}}_{s}$ because it does not satisfy equation (1.7). To show our claim,

$$
\begin{aligned}
d_{2}\left(\chi_{K_{n}}, \chi_{K}\right)^{2} & =\int_{0}^{\infty}\left|\chi_{\left(\frac{\pi}{2}, \frac{2^{n} \pi}{2^{n+1}-1}\right)}\right|^{2} \stackrel{d \xi}{\xi}+\int_{0}^{\infty}\left|\chi_{\left[2^{n} \pi, 2^{n} \pi+\frac{2^{n} \pi}{2^{n+1}-1}\right.}\right|^{2} \stackrel{d \xi}{\xi} \\
& =\ln \left(\frac{2^{n+1}}{2^{n+1}-1}\right)+\ln \left(1+\frac{1}{2^{n+1}-1}\right) \rightarrow 0, \quad \text { as } n \rightarrow \infty .
\end{aligned}
$$

In particular, this implies that $\left\{\hat{\psi}_{n}\right\}_{n=1}^{\infty}$ is $d_{2}$-Cauchy, but it does not converge to a function in $\widehat{\mathcal{W}}$. Therefore, $\left(\widehat{\mathcal{W}}, d_{2}\right)$ is not complete. Note that, in view of Theorem 1.13, the sequence $\left\{\hat{\psi}_{n}\right\}_{n=1}^{\infty}$ cannot be $d_{1}$-Cauchy.

\section{THE PROOF OF THE THEOREM}

In this section, we prove a slightly more general version of Theorem 1.13. We consider a set $F \subset L^{2}(\mathbb{R}, d \xi) \cap L^{2}\left(\mathbb{R}_{*},|\xi \xi|\right)$ which contains $\widehat{\mathcal{W}}$ as a proper closed subset. We then show that $(F, d)$ is complete and that, in $F$, the metrics $d, d_{1}$ and $d_{2}$ are equivalent.

Define

$$
F=\left\{f \in L^{2}(\mathbb{R}, d \xi) \cap L^{2}\left(\mathbb{R}_{*}, \mid \xi \xi\right): \delta_{f}(\xi)=\tau_{f}(\xi)=1, \text { a.e. } \xi \in \mathbb{R}\right\},
$$

where $\delta_{f}, \tau_{f}$ are given by (1.5) and (1.7). Note that, if $f \in F$ and $g$ is any (measurable) function on $\mathbb{R}$ with $|f|=|g|$ a.e., then $g \in F$. Note also that $\widehat{\mathcal{W}} \subset F$ (because of (1.5) and (1.7)), but $\widehat{\mathcal{W}} \neq F$. One way of seeing this is to note that if $\hat{\psi} \in \widehat{\mathcal{W}}$, then not every other function $\hat{\eta}$ with $|\hat{\eta}|=|\hat{\psi}|$ is the Fourier transform of a wavelet. In fact, $|\hat{\psi}| \in \widehat{\mathcal{W}}$ if and only if $|\hat{\psi}|$ is the characteristic function of a set; that is, if 
$|\hat{\psi}| \in \widehat{\mathcal{W}}_{s}$. A proof of this property is presented in the appendix, together with a less trivial example of a function $\hat{\psi} \in F \backslash \widehat{\mathcal{W}}$.

Our main result in this section is the following:

Theorem 2.2. In the set $F$, defined in (2.1), the metrics $d, d_{1}$ and $d_{2}$ are all topologically equivalent. Moreover, $(F, d)$ is a complete metric space.

Before embarking on the proof of Theorem 2.2, we show how to obtain Theorem 1.13 from Theorem 2.2:

Corollary 2.3. The space $(\widehat{\mathcal{W}}, d)$ is a closed subspace of $(F, d)$. In particular, $(\widehat{\mathcal{W}}, d)$ is a complete metric space.

Proof. Suppose $\left\{\hat{\psi}_{n}\right\}_{n=1}^{\infty} \subset \widehat{\mathcal{W}}$ is a $d$-Cauchy sequence. Then, it follows from Theorem 2.2 that there is a $\hat{\psi} \in F$ such that $d\left(\hat{\psi}_{n}, \hat{\psi}\right) \rightarrow 0$, and in particular, $\hat{\psi}_{n} \rightarrow \hat{\psi}$ in the norm of $L^{2}(\mathbb{R}, d \xi)$. On the other hand, the fact that $\hat{\psi} \in F$ implies that (1.5) holds and (from (1.7)) that $\|\hat{\psi}\|_{L^{2}(\mathbb{R}, d \xi)}^{2}=2 \pi$. Hence, to show that $\hat{\psi} \in \widehat{\mathcal{W}}$, it suffices to check that (1.6) holds; that is, $t_{q, \hat{\psi}}=0$ for all $q \in 2 \mathbb{Z}+1$.

Now, since $\hat{\psi}_{n} \in \widehat{\mathcal{W}}$, we have that $t_{q, \hat{\psi}_{n}}=0$ for all $q \in 2 \mathbb{Z}+1$, and therefore,

$$
\begin{aligned}
\int_{\mathbb{R}} \mid & t_{q, \hat{\psi}}(\xi)\left|d \xi=\int_{\mathbb{R}}\right| t_{q, \hat{\psi}}(\xi)-t_{q, \hat{\psi}_{n}}(\xi) \mid d \xi \\
\leq & \int_{\mathbb{R}} \sum_{j=0}^{\infty}\left|\left(\hat{\psi}-\hat{\psi}_{n}\right)\left(2^{j} \xi\right)\right|\left|\hat{\psi}\left(2^{j}(\xi+2 \pi q)\right)\right| d \xi \\
& +\int_{\mathbb{R}} \sum_{j=0}^{\infty}\left|\hat{\psi}_{n}\left(2^{j} \xi\right)\right|\left|\left(\hat{\psi}-\hat{\psi}_{n}\right)\left(2^{j}(\xi+2 q \pi)\right)\right| d \xi \\
\leq & \sum_{j=0}^{\infty}\left(\int_{\mathbb{R}}\left|\left(\hat{\psi}-\hat{\psi}_{n}\right)\left(2^{j} \xi\right)\right|^{2} d \xi\right)^{1 / 2}\left(\int_{\mathbb{R}}\left|\hat{\psi}\left(2^{j}(\xi+2 q \pi)\right)\right|^{2} d \xi\right)^{1 / 2} \\
& +\sum_{j=0}^{\infty}\left(\int_{\mathbb{R}}\left|\hat{\psi}_{n}\left(2^{j} \xi\right)\right|^{2} d \xi\right)^{1 / 2}\left(\int_{\mathbb{R}}\left|\left(\hat{\psi}-\hat{\psi}_{n}\right)\left(2^{j}(\xi+2 q \pi)\right)\right|^{2} d \xi\right)^{1 / 2} \\
\leq & \sum_{j=0}^{\infty} 2^{-j}\left\|\hat{\psi}-\hat{\psi}_{n}\right\|_{L^{2}(\mathbb{R}, d \xi)}\|\hat{\psi}\|_{L^{2}(\mathbb{R}, d \xi)}+\sum_{j=0}^{\infty} 2^{-j}\left\|\hat{\psi}_{n}\right\|_{L^{2}(\mathbb{R}, d \xi)}\left\|\hat{\psi}-\hat{\psi}_{n}\right\|_{L^{2}(\mathbb{R}, d \xi)} \\
= & 4 \sqrt{2 \pi}\left\|\hat{\psi}-\hat{\psi}_{n}\right\|_{L^{2}(\mathbb{R}, d \xi)},
\end{aligned}
$$

which goes to 0 as $n \rightarrow \infty$. Thus, $t_{q, \hat{\psi}}=0$ (a.e.) and (1.6) holds. This establishes the corollary.

The following corollary is an even simpler consequence of Theorem 2.2.

Corollary 2.4. The set $\widehat{\mathcal{W}}_{s}$ is $d$-closed in $L^{2}(\mathbb{R}, d \xi) \cap L^{2}\left(\mathbb{R}_{*},|\xi \xi|\right)$.

Proof. Since the elements of $\widehat{\mathcal{W}}_{s}$ are all characteristic functions of sets, the limit point of any $d$-Cauchy sequence in $\widehat{\mathcal{W}}_{s}$ must be a characteristic function of a set as well (by the a.e. convergence of a subsequence). On the other hand, by Theorem 2.2, this limit point must also belong to $F$, and therefore, in view of (1.5) and (1.7), it must be the Fourier transform of a wavelet. Thus, it belongs to $\widehat{\mathcal{W}}_{s}$ and $\widehat{\mathcal{W}}_{s}$ is closed. 
We conclude this section by proving Theorem 2.2 :

Proof of Theorem 2.2. Part 1. We show that in $F, d$ and $d_{1}$ are equivalent metrics. It is enough to see that any sequence $\left(f_{n}\right)_{n=1}^{\infty} \subset F$ which converges to a function $f \in F$ in the metric $d_{1}$ also converges to $f$ in the metric $d_{2}$. Indeed, this would show that the identity map $I:\left(F, d_{1}\right) \rightarrow(F, d)$ is a homeomorphism.

Suppose then that $f_{n}, f \in F, n=1,2, \ldots$, and that $\lim _{n \rightarrow \infty} d_{1}\left(f_{n}, f\right)=0$. Then,

$$
\begin{aligned}
d_{2}\left(f_{n}, f\right)^{2} & =\int_{\mathbb{R}_{*}}\left|\left(f_{n}-f\right)(\xi)\right|^{2} \frac{d \xi}{|\xi|} \\
& =\int_{\mathbb{R}_{*}}\left(f_{n}-f\right)(\xi) \overline{\left(f_{n}-f\right)(\xi)} \frac{d \xi}{|\xi|} \\
& =\int_{\mathbb{R}_{*}}\left|f_{n}(\xi)\right|^{2} \frac{d \xi}{|\xi|}+\int_{\mathbb{R}_{*}}|f(\xi)|^{2} \frac{d \xi}{|\xi|}-2 \int_{\mathbb{R}_{*}} \Re e\left[f(\xi) \overline{f_{n}(\xi)} \frac{d \xi}{|\xi|}\right.
\end{aligned}
$$

Now, since $f_{n}, f \in F$, we have that

$$
\int_{\mathbb{R}_{*}}|f(\xi)|^{2} \frac{d \xi}{|\xi|}=\int_{\mathbb{R}_{*}}\left|f_{n}(\xi)\right|^{2} \frac{d \xi}{|\xi|}=\ln (4) .
$$

Thus,

$$
\begin{aligned}
d_{2}\left(f_{n}, f\right)^{2} & =2\left(\int_{\mathbb{R}_{*}}|f(\xi)|^{2} \frac{d \xi}{|\xi|}-\int_{\mathbb{R}_{*}} \Re e\left[f(\xi) \overline{f_{n}(\xi)}\right] \frac{d \xi}{|\xi|}\right) \\
& =2 \Re e\left(\int_{\mathbb{R}_{*}} f(\xi) \overline{\left(f(\xi)-f_{n}(\xi)\right)} \frac{d \xi}{|\xi|}\right) .
\end{aligned}
$$

We want to show that the last term above goes to 0 as $n \rightarrow \infty$. Let $\epsilon>0$, and choose $\delta=\delta(\epsilon)>0$ such that $\int_{|\xi|<\delta}|f(\xi)|^{2} \frac{d \xi}{|\xi|}<\epsilon^{2}$. Choose $n_{0}=n_{0}(\delta, \epsilon) \in \mathbb{N}$ such that, for $n \geq n_{0}$, we have

$$
d_{1}\left(f, f_{n}\right)^{2}=\int_{\mathbb{R}}\left|f(\xi)-f_{n}(\xi)\right|^{2} d \xi<\delta \epsilon^{2} .
$$

Then, we have

$$
\begin{aligned}
d_{2}\left(f_{n}, f\right) \leq & 2\left(\int_{|\xi|<\delta}|f(\xi)|\left|f(\xi)-f_{n}(\xi)\right| \frac{d \xi}{|\xi|}+\int_{|\delta|>\delta}|f(\xi)|\left|\left(f-f_{n}\right)(\xi)\right| \frac{d \xi}{|\xi|}\right) \\
\leq & 2\left(\int_{|\xi|<\delta}|f(\xi)|^{2} \frac{d \xi}{|\xi|}\right)^{1 / 2}\left(\int_{\mathbb{R}}\left|\left(f-f_{n}\right)(\xi)\right|^{2} \frac{d \xi}{|\xi|}\right)^{1 / 2} \\
& +2\left(\int_{\mathbb{R}}|f(\xi)|^{2} \frac{d \xi}{|\xi|}\right)^{1 / 2}\left(\int_{|\xi|>\delta}\left|f(\xi)-f_{n}(\xi)\right|^{2} \frac{d \xi}{|\xi|}\right)^{1 / 2} \\
< & 2 \epsilon\left(\|f\|_{L^{2}\left(\mathbb{R}_{*},|\xi|\right)}^{d \xi}+\left\|f_{n}\right\|_{L^{2}\left(\mathbb{R}_{*},|\xi|\right)}^{d \xi}\right)+2\|f\|_{L^{2}\left(\mathbb{R}_{*},|\xi|\right)} \frac{\sqrt{\delta} \epsilon}{\sqrt{\delta}} \\
= & 6 \sqrt{\ln (4)} \epsilon .
\end{aligned}
$$

Thus, $\lim _{n \rightarrow \infty} d_{2}\left(f_{n}, f\right)=0$, and part 1 is established. 
Part 2. We show that $d$ and $d_{2}$ are equivalent metrics in $F$. As in part 1, it suffices to show that, if $f_{n}, f \in F$ for $n=1,2, \ldots$ and $\lim _{n \rightarrow \infty} d_{2}\left(f_{n}, f\right)=0$, then $\lim _{n \rightarrow \infty} d_{1}\left(f_{n}, f\right)=0$. Indeed, this implies that $I:\left(F, d_{2}\right) \rightarrow(F, d)$ is a homeomorphism, and therefore, that the metrics $d_{2}$ and $d$ are equivalent on $F$.

Let $\epsilon>0$ and choose $M=M(\epsilon)>0$ such that $\int_{|\xi|>M}|f(\xi)|^{2} d \xi<\epsilon^{2}$. Choose $n_{0}=n_{0}(\epsilon, M)$ such that

$$
d_{2}\left(f, f_{n}\right)^{2}=\int_{\mathbb{R}_{*}}\left|\left(f-f_{n}\right)(\xi)\right|^{2} \frac{d \xi}{|\xi|}<\frac{\epsilon^{2}}{M}
$$

for $n \geq n_{0}$. Then, for any such $n$, we have

$$
\begin{aligned}
d_{1}\left(f, f_{n}\right)^{2} & =\int_{\mathbb{R}}\left|\left(f-f_{n}\right)(\xi)\right|^{2} d \xi \\
& =\int_{\mathbb{R}}|f(\xi)|^{2} d \xi+\int_{\mathbb{R}}\left|f_{n}(\xi)\right|^{2} d \xi-2 \int_{\mathbb{R}} \Re e\left[f(\xi) \overline{f_{n}(\xi)}\right] d \xi \\
& =2 \Re e\left[\int_{\mathbb{R}} f(\xi) \overline{\left(f-f_{n}\right)(\xi)} d \xi\right],
\end{aligned}
$$

where in the last equality we have used that, for $f, f_{n} \in F$,

$$
\int_{\mathbb{R}}|f(\xi)|^{2} d \xi=\int_{\mathbb{R}}\left|f_{n}(\xi)\right|^{2} d \xi=2 \pi
$$

Now, separating the last integral into $|\xi| \leq M$ and $|\xi| \geq M$ and using CauchySchwarz's inequality in each new integral, we obtain

$$
\begin{aligned}
d_{1}\left(f, f_{n}\right)^{2} \leq & 2\left(\int_{|\xi| \leq M}|f(\xi)|^{2} d \xi\right)^{1 / 2}\left(\int_{|\xi| \leq M}\left|\left(f-f_{n}\right)(\xi)\right|^{2} d \xi\right)^{1 / 2} \\
& +2\left(\int_{|\xi|>M}|f(\xi)|^{2} d \xi\right)^{1 / 2}\left(\int_{|\xi|>M}\left|\left(f-f_{n}\right)(\xi)\right|^{2} d \xi\right)^{1 / 2} \\
\leq & 2 \sqrt{2 \pi}\left(\int_{|\xi| \leq M}\left|\left(f-f_{n}\right)(\xi)\right|^{2} \frac{d \xi}{|\xi|}\right)^{1 / 2} M^{1 / 2} \\
& +2 \epsilon\left(\|f\|_{L^{2}(\mathbb{R}, d \xi)}+\left\|f_{n}\right\|_{L^{2}(\mathbb{R}, d \xi)}\right) \\
< & 6 \sqrt{2 \pi} \epsilon .
\end{aligned}
$$

This concludes part 2 .

Part 3. To conclude the proof of the theorem, we show that $(F, d)$ is complete. Suppose that $\left(f_{n}\right)_{n=1}^{\infty} \subset F$ is a $d$-Cauchy sequence. Then, by completeness of the Hilbert spaces $L^{2}(\mathbb{R}, d \xi)$ and $L^{2}\left(\mathbb{R}_{*},|\xi \xi|\right)$, there is a function $f \in L^{2}(\mathbb{R}, d \xi) \cap$ $L^{2}\left(\mathbb{R}_{*}, \mid \xi \xi\right)$ such that $\lim _{n \rightarrow \infty} d\left(f_{n}, f\right)=0$. We shall show that $f \in F$. Indeed, let us first verify that $f$ satisfies equation $(1.7)$; that is, $\tau_{f}(\xi)=1$, a.e. 
Given $\ell \in \mathbb{Z}$, we have

$$
\begin{aligned}
\int_{-\pi}^{\pi}\left(\sum_{k \in \mathbb{Z}}|f(\xi+2 \pi k)|^{2}\right) e^{i \ell \xi} d \xi & =\int_{\mathbb{R}}|f(\xi)|^{2} e^{i \ell \xi} d \xi \\
& =\lim _{n \rightarrow \infty} \int_{\mathbb{R}}\left|f_{n}(\xi)\right|^{2} e^{i \ell \xi} d \xi \\
& =\lim _{n \rightarrow \infty} \int_{-\pi}^{\pi}\left(\sum_{k \in \mathbb{Z}}\left|f_{n}(\xi+2 k \pi)\right|^{2}\right) e^{i \ell \xi} d \xi \\
& =2 \pi \delta_{0, \ell} .
\end{aligned}
$$

Here, the pass to the limit in the second equality can be justified by using CauchySchwarz's inequality. Indeed, for $\ell \in \mathbb{Z}$,

$$
\begin{aligned}
\int_{\mathbb{R}}\left(|f(\xi)|^{2}-\left|f_{n}(\xi)\right|^{2}\right) e^{i \ell \xi} d \xi & \leq \int_{R}|| f(\xi)|-| f_{n}(\xi)||\left(|f(\xi)|+\left|f_{n}(\xi)\right|\right) d \xi \\
& \leq\left(\int_{\mathbb{R}}\left|\left(f-f_{n}\right)(\xi)\right|^{2} d \xi\right)^{\frac{1}{2}}\left(\int_{\mathbb{R}}\left(|f(\xi)|+\left|f_{n}(\xi)\right|\right)^{2} d \xi\right)^{\frac{1}{2}} \\
& \leq d_{1}\left(f_{n}, f\right)\left(\|f\|_{L^{2}(\mathbb{R}, d \xi)}+\left\|f_{n}\right\|_{L^{2}(\mathbb{R}, d \xi)}\right) \rightarrow 0
\end{aligned}
$$

as $n \rightarrow \infty$. Clearly, (2.5) implies that $\sum_{k \in \mathbb{Z}}|f(\xi+2 k \pi)|^{2}=1$ a.e., and (1.7) holds for $f$.

Similarly, we prove that (1.5) holds by finding the "Fourier coefficients" of $\delta_{f}(\xi)=\sum_{j \in \mathbb{Z}}\left|f\left(2^{j} \xi\right)\right|^{2}$ in $L^{2}([1,2], \stackrel{d \xi}{\xi})$. To be more precise, let $\Delta_{f}(\xi):=\delta_{f}\left(2^{\xi}\right)=$ $\sum_{j \in \mathbb{Z}}\left|f\left(2^{j+\xi}\right)\right|^{2}$, if $\xi \in \mathbb{R}$. Clearly, $\Delta_{f}$ is 1-periodic.

To prove $\delta_{f}(\xi)=1$, a.e. on $\mathbb{R}^{+}$, it is enough to see that, for $\ell \in \mathbb{Z}$,

$$
\int_{0}^{1} \Delta_{f}(\xi) e^{2 \pi i \ell \xi} d \xi=\delta_{\ell, 0}
$$

Indeed, by changing variables $\eta=2^{\xi}$, we have

$$
\begin{aligned}
\int_{0}^{1} \Delta_{f}(\xi) e^{2 \pi i \ell \xi} d \xi & =\int_{1}^{2} \delta_{f}(\eta) e^{2 \pi i \ell \frac{\ln \eta}{\ln 2}} \frac{d \eta}{(\ln 2) \eta} \\
& =\sum_{j \in \mathbb{Z}} \int_{1}^{2}\left|f\left(2^{j} \eta\right)\right|^{2} e^{2 \pi i \ell \frac{\ln \eta}{\ln 2}} \frac{d \eta}{(\ln 2) \eta} \\
& =\sum_{j \in \mathbb{Z}} \int_{2^{j}}^{2^{j+1}}|f(\xi)|^{2} e^{2 \pi i \ell \frac{\ln \xi}{\ln 2}} \frac{d \xi}{(\ln 2) \xi} \\
& =\int_{0}^{\infty}|f(\xi)|^{2} e^{2 \pi i \ell \frac{\ln \xi}{\ln 2}} \frac{d \xi}{(\ln 2) \xi} \\
& =\lim _{n \rightarrow \infty} \int_{0}^{\infty}\left|f_{n}(\xi)\right|^{2} e^{2 \pi i \ell \frac{\ln \xi}{\ln 2}} \frac{d \xi}{(\ln 2) \xi} \\
& =\lim _{n \rightarrow \infty} \int_{0}^{1} \Delta_{f_{n}}(\xi) e^{2 \pi i \ell \xi} d \xi \\
& =\delta_{0, \ell},
\end{aligned}
$$


where the second to last equality is justified by repeating the first four steps in reverse, and the equality which allows us to pass to the limit above can be justified using Cauchy-Schwarz in the same way as before. This establishes (2.6).

In a similar way, one can show that $\delta_{f}(\xi)=1$ holds for (a.e.) $\xi<0$ by replacing $f, f_{n}$ with $\tilde{f}, \tilde{f}_{n}$, where $\tilde{f}(\xi)=f(-\xi)$. This would establish (1.5), which together with (1.7) implies that $f \in F$ and $F$ is $d$-complete.

\section{Appendix}

We claimed in section 2 that the only wavelets with positive Fourier transform are those in $\mathcal{W}_{s}$. Indeed, suppose $\psi$ is a wavelet such that $\hat{\psi} \geq 0$. Then, for every fixed $\xi \in \mathbb{R}$, such that $\hat{\psi}(\xi) \neq 0$, the equations in (1.8) imply that $\hat{\psi}\left(2^{j} \xi\right)=0, \forall j \geq 1$. Then, if $\hat{\psi}(\xi)<1$, there must be a $j_{0}<0$ such that $\hat{\psi}\left(2^{j_{0}} \xi\right) \neq 0$ (by (1.5)) but, again, the equations in (1.8) would imply that $\hat{\psi}\left(2^{j_{0}+k} \xi\right)=0, \forall k \geq 1$, which is a contradiction. Thus, $\hat{\psi}(\xi)=1$ and $\hat{\psi}$ is the characteristic function of a set.

Finally, we give a particularly interesting example of a function $\hat{\psi} \in F \backslash \mathcal{W}$. More precisely, we construct a non-negative $\hat{\psi} \in F$ so that there is no real-valued function $\alpha: \mathbb{R} \rightarrow \mathbb{R}$ that makes $e^{i \alpha} \hat{\psi}$ into the Fourier transform of a wavelet. In a sense, this example shows the independence of (1.5) and (1.7), from the equations in (1.6), at least when the measure of the support of $\hat{\psi}$ is large enough.

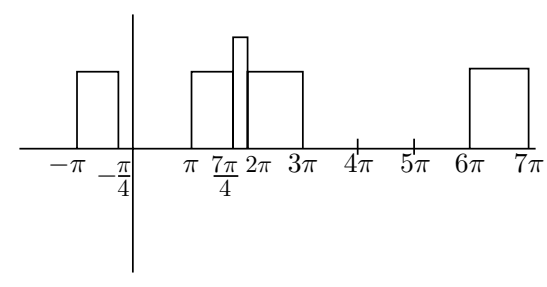

DiAGRAM 2.

Example 3. Define $\hat{\psi}$ as follows (see Diagram 2):

$$
\hat{\psi}(\xi)= \begin{cases}\frac{1}{\sqrt{2}}, & \text { if } \xi \in\left(-\pi, \frac{-\pi}{4}\right] \cup\left(\pi, \frac{7 \pi}{4}\right] \cup(2 \pi, 3 \pi] \cup(6 \pi, 7 \pi], \\ 1, & \text { if } \xi \in\left(\frac{7 \pi}{4}, 2 \pi\right], \\ 0, & \text { elsewhere. }\end{cases}
$$

It is easy to check that $\hat{\psi}$ satisfies (1.5) and (1.7). However, $\left(e^{i \alpha} \hat{\psi}\right)^{\swarrow}$ can never be a wavelet because

$$
t_{-1}(\xi)=\frac{1}{2} e^{i(\alpha(\xi)-\alpha(\xi-2 \pi))} \neq 0, \quad \text { if } \xi \in\left(\pi, \frac{3 \pi}{2}\right] .
$$

\section{REFERENCES}

[FW] Fang, X. and Wang, X., Construction of minimally supported frequency wavelets, J. Fourier Anal. Appl 24 (1996), 315-328. MR 97d:42030

[HKLS] Y-H. Ha, H. Kang, J. Lee and J. Seo, Unimodular wavelets for $L^{2}$ and the Hardy space $H^{2}$, Michigan Math. J. 41 (1994), 345-361. MR 95g:42050 
[HW] Hernández, E. and Weiss, G. L., A First Course on Wavelets, CRC Press, 1996. MR 97i:42015

[SPEE] D. M. Speegle, The s-elementary wavelets are path connected, Proc. AMS 127 (1999), no. 1, 223-233. MR 99b:42045

Department of Mathematics, Washington University, Saint Louis, Missouri 63130

E-mail address: gustavo@math.wustl.edu

Current address: Dipartimento di Matematica, Università di Milano, Via C. Saldini, 50, 20133, Milano, Italy

E-mail address: gustavo@ares.mat.unimi.it

Department of Mathematics, Saint Louis University, Saint Louis, Missouri 63103

E-mail address: speegled@slu.edu 